\title{
Nontrivial solutions for boundary value problems of a fourth order difference equation with sign-changing nonlinearity
}

\section{Keyu Zhang ${ }^{1 *}$, Donal O'Regan ${ }^{2}$ and Zhengqing $\mathrm{Fu}^{3}$}

"Correspondence:

keyu_292@163.com

1School of Mathematics, Qilu

Normal University, Jinan, China Full list of author information is

available at the end of the article

\begin{abstract}
In this paper, using the topological degree theory, we establish two existence theorems for nontrivial solutions for boundary value problems of a fourth order difference equation with a sign-changing nonlinearity.
\end{abstract}

Keywords: Difference equations boundary value problems; Sign-changing nonlinearity; Nontrivial solutions; Topological degree theory

\section{Introduction}

For $a, b \in \mathbb{Z}$, let $\mathbb{T}_{a}^{b}=\{a, a+1, a+2, \ldots, b\}$ with $a<b$. In this paper we consider the existence of nontrivial solutions for boundary value problems of the following fourth order difference equation with a sign-changing nonlinearity

$$
\left\{\begin{array}{l}
\Delta^{4} u(t-2)=f(t, u(t)) \\
u(1)=u(T+1)=\Delta^{2} u(0)=\Delta^{2} u(T)=0
\end{array}\right.
$$

where $T$ is an integer with $T \geq 5$, and $f: \mathbb{T}_{2}^{T} \times \mathbb{R} \rightarrow \mathbb{R}$ is a continuous function with $\mathbb{T}_{2}^{T}=\{2,3, \ldots, T\}$ and $\mathbb{R}=(-\infty,+\infty)$ (it is assumed to be continuous from the topological space $\mathbb{T}_{2}^{T} \times \mathbb{R}$ into the topological space $\mathbb{R}$, the topology on $\mathbb{T}_{2}^{T}$ being the discrete topology).

Difference equations with discrete boundary value conditions have been widely studied in the literature; see, for example, [1-11] and the references therein. However, as mentioned in [6], very few results are available with sign-changing nonlinearities; see [6-11]. Other related work in this field can be found in [12-45] and the references therein. In [7], C.S. Goodrich used the Krasnosel'skii fixed point theorem to obtain the existence of at least one positive solution to the following discrete fractional semipositone boundary value problem

$$
\left\{\begin{array}{l}
\Delta^{v} y(t)=\lambda f(t+v-1, y(t+v-1)), \quad t \in[0, T] \cap \mathbb{Z}, \\
y(v-1)=y(v+T)+\sum_{i=1}^{N} F\left(t_{i}, y\left(t_{i}\right)\right)
\end{array}\right.
$$

(c) The Author(s) 2018. This article is distributed under the terms of the Creative Commons Attribution 4.0 International License (http://creativecommons.org/licenses/by/4.0/), which permits unrestricted use, distribution, and reproduction in any medium, provided you give appropriate credit to the original author(s) and the source, provide a link to the Creative Commons license, and indicate if changes were made. 
where $\Delta^{v}$ is the $v$ th fractional difference with $v \in(0,1), f$ is continuous, bounded below (i.e., $f+M \geq 0$ for some $M>0$ ), and

$$
\lim _{y \rightarrow+\infty} \frac{f(t, y)}{y}=0 \quad \text { uniformly for } t \in[v-1, v+T]_{\mathbb{Z}_{v-1}} .
$$

In [10], J. Xu and D. O'Regan used the fixed point index to obtain the existence of nontrivial solutions for (1.2) with weaker conditions than that of (1.3), and also in [11], J. Xu et al. considered the existence of positive solutions for system (1.2), with adopted convex and concave functions to depict the coupling behavior of nonlinearities. In [40], Y. Cui used the $u_{0}$-positive operator to study the uniqueness of solutions for the following nonlinear fractional boundary value problems:

$$
\left\{\begin{array}{l}
D^{p} x(t)+p(t) f(t, x(t))+q(t)=0, \quad t \in(0,1), \\
x(0)=x^{\prime}(0)=0, \quad x(1)=0,
\end{array}\right.
$$

where $D^{p}$ is the Riemann-Liouville fractional derivative, and $f$ is a Lipschitz continuous function, with the Lipschitz constant associated with the first eigenvalue for the relevant operator. Using similar methods, the authors in $[12,39,41]$ obtained some existence and nonexistence theorems for their problems.

Motivated by the works mentioned above, we consider the existence of nontrivial solutions for (1.1) involving sign-changing nonlinearities. Using the topological degree theory of a completely continuous field, and conditions concerning the first eigenvalue corresponding to the relevant linear problem, two existence theorems are obtained.

\section{Preliminaries}

For convenience, we let $\mathbb{T}_{1}^{T+1}=\{1,2,3, \ldots, T, T+1\}, \mathbb{T}_{0}^{T+2}=\{0,1,2,3, \ldots, T+1, T+2\}$, $\mathbb{T}_{2}^{T}=\{2,3, \ldots, T\}$. Then we define our space $E$ as the collection of all maps from $\mathbb{T}_{0}^{T+2}$ to $\mathbb{R}$ equipped with the norm $\|u\|=\max _{j \in \mathbb{T}_{0}^{T+2}}|u(j)|$. Consequently, $E$ is a Banach space, and we let $P=\left\{u \in E: u(t) \geq 0, t \in \mathbb{T}_{1}^{T+1}\right\}$. Then $P$ is a cone on $E$. Throughout our paper, we let $B_{\rho}=\{u \in E:\|u\|<\rho\}$ for $\rho>0$. Now $\partial B_{\rho}=\{u \in E:\|u\|=\rho\}$ and $\bar{B}_{\rho}=\{u \in E:\|u\| \leq \rho\}$.

In what follows, we establish the Green's function for (1.1). As in [3, 4], we transform (1.1) into its equivalent sum equation

$$
u(t)=\sum_{s=2}^{T} H(t, s) \sum_{j=2}^{T} H(s, j) f(j, u(j)), \quad t \in \mathbb{T}_{1}^{T+1},
$$

where

$$
H(t, s)=\frac{1}{T} \begin{cases}(t-1)(T+1-s), & 1 \leq t \leq s \leq T \\ (s-1)(T+1-t), & 2 \leq s \leq t \leq T+1\end{cases}
$$

Lemma 2.1 Green's function $H$ has the following properties:

(i) $H(t, s)>0$ for $(t, s) \in \mathbb{T}_{2}^{T} \times \mathbb{T}_{2}^{T}$,

(ii) $\frac{1}{T} H(t, t) H(s, s) \leq H(t, s) \leq H(s, s)$ for $(t, s) \in \mathbb{T}_{2}^{T} \times \mathbb{T}_{1}^{T+1}$. 
Proof We only need to prove the first inequality of (ii). Indeed, for all $(t, s) \in \mathbb{T}_{2}^{T} \times \mathbb{T}_{1}^{T+1}$, from the definitions of $H(t, s)$ and $H(s, s)$ we have

$$
\frac{H(t, s)}{H(s, s)}= \begin{cases}\frac{t-1}{s-1} \geq \frac{t-1}{T} \geq \frac{t-1}{T} \frac{T+1-t}{T}=\frac{1}{T} H(t, t), & 1 \leq t \leq s \leq T \\ \frac{T+1-t}{T+1-s} \geq \frac{T+1-t}{T} \geq \frac{T+1-t}{T} \frac{t-1}{T}=\frac{1}{T} H(t, t), & 2 \leq s \leq t \leq T+1\end{cases}
$$

Then we have $H(t, s) \geq \frac{1}{T} H(t, t) H(s, s)$ for $(t, s) \in \mathbb{T}_{2}^{T} \times \mathbb{T}_{1}^{T+1}$. This completes the proof.

We define an operator $A: E \rightarrow E$ as follows:

$$
(A u)(t)=\sum_{s=2}^{T} H(t, s) \sum_{j=2}^{T} H(s, j) f(j, u(j)), \quad t \in \mathbb{T}_{1}^{T+1}
$$

The existence of solutions for (1.1) is equivalent to that of fixed points of $A$.

From [4], we know that $\sin \frac{\pi(t-1)}{T}:=\varphi_{0}(t), t \in \mathbb{T}_{2}^{T}$ is the eigenfunction related to the eigenvalue $\frac{1}{16} \sin ^{-4} \frac{\pi}{2 T}$ of the eigenproblem

$$
\left\{\begin{array}{l}
\Delta^{4} u(t-2)=\lambda u(t), \quad t \in \mathbb{T}_{2}^{T}, \\
u(1)=u(T+1)=\Delta^{2} u(0)=\Delta^{2} u(T)=0
\end{array}\right.
$$

i.e., the following two equations hold:

$$
\begin{aligned}
& \sum_{s=2}^{T} \sum_{j=2}^{T} H(t, s) H(s, j) \sin \frac{\pi(j-1)}{T}=\frac{1}{16} \sin ^{-4} \frac{\pi}{2 T} \sin \frac{\pi(t-1)}{T}, \quad t \in \mathbb{T}_{2}^{T}, \\
& \sum_{s=2}^{T} \sum_{t=2}^{T} H(t, s) H(s, j) \sin \frac{\pi(t-1)}{T}=\frac{1}{16} \sin ^{-4} \frac{\pi}{2 T} \sin \frac{\pi(j-1)}{T}, \quad t \in \mathbb{T}_{2}^{T} .
\end{aligned}
$$

Lemma 2.2 Let $e(t)=\frac{1}{T} H(t, t)$ and $P_{0}=\left\{u \in P: u(t) \geq e(t)\|u\|, t \in \mathbb{T}_{1}^{T+1}\right\}$. Then $L(P) \subset P_{0}$, where

$$
(L u)(t)=\sum_{s=2}^{T} H(t, s) \sum_{j=2}^{T} H(s, j) u(j), \quad t \in \mathbb{T}_{1}^{T+1}
$$

This is a direct result from Lemma 2.1(ii), so we omit its proof.

Now, we offer two basic theorems from the topological degree theory; for details we refer the reader to [46].

Lemma 2.3 Let E be a Banach space and $\Omega$ a bounded open set in E. Suppose that $A: \Omega \rightarrow$ $E$ is a continuous compact operator. If there exists $u_{0} \in E \backslash\{0\}$ such that

$$
u-A u \neq \mu u_{0}, \quad \forall u \in \partial \Omega, \mu \geq 0,
$$

then the topological degree $\operatorname{deg}(I-A, \Omega, 0)=0$. 
Lemma 2.4 Let $E$ be a Banach space and $\Omega$ a bounded open set in $E$ with $0 \in \Omega$. Suppose that $A: \Omega \rightarrow E$ is a continuous compact operator. If

$A u \neq \mu u, \quad \forall u \in \partial \Omega, \mu \geq 1$,

then the topological degree $\operatorname{deg}(I-A, \Omega, 0)=1$.

\section{Nontrivial solutions for (1.1)}

Now we present some assumptions for our nonlinearity $f$.

(H1) There exist two constants $a>0, b>0$ and a function $k \in C\left(\mathbb{R}, \mathbb{R}^{+}\right)$such that

$$
f(t, u) \geq-a-b k(u), \quad \forall u \in \mathbb{R}, t \in \mathbb{T}_{2}^{T} .
$$

(H2) $\lim _{|u| \rightarrow+\infty} \frac{k(u)}{|u|}=0$.

(H3) $\liminf |u| \rightarrow+\infty \frac{f(t, u)}{|u|}>16 \sin ^{4} \frac{\pi}{2 T}$ uniformly on $t \in \mathbb{T}_{2}^{T}$,

(H4) $\lim \sup _{|u| \rightarrow 0} \frac{|f(t, u)|}{|u|}<16 \sin ^{4} \frac{\pi}{2 T}$ uniformly on $t \in \mathbb{T}_{2}^{T}$,

(H5) $\liminf _{u \rightarrow 0^{+}} \frac{f(t, u)}{u}>16 \sin ^{4} \frac{\pi}{2 T}, \limsup _{u \rightarrow 0^{-}} \frac{f(t, u)}{u}<16 \sin ^{4} \frac{\pi}{2 T}$, uniformly on $t \in \mathbb{T}_{2}^{T}$,

(H6) $\lim \sup _{|u| \rightarrow+\infty} \frac{|f(t, u)|}{|u|}<16 \sin ^{4} \frac{\pi}{2 T}$ uniformly on $t \in \mathbb{T}_{2}^{T}$.

Theorem 3.1 Suppose that (H1)-(H4) hold. Then (1.1) has at least one nontrivial solution.

Proof From (H3) there exist $\varepsilon_{0}>0$ and $X_{0}>0$ such that

$$
f(t, u) \geq\left(16 \sin ^{4} \frac{\pi}{2 T}+\varepsilon_{0}\right)|u|, \quad \forall t \in \mathbb{T}_{2}^{T},|u|>X_{0}
$$

For any given $\varepsilon$ with $\varepsilon_{0}-b \varepsilon>0$, and from (H2), there exists $X_{1}>X_{0}$ such that

$$
k(u) \leq \varepsilon|u|, \quad \forall|u|>X_{1} .
$$

Now since $a>0, b>0$ and $k$ is a nonnegative function, we have

$$
\begin{aligned}
f(t, u) & \geq\left(16 \sin ^{4} \frac{\pi}{2 T}+\varepsilon_{0}\right)|u|-a-b k(u) \\
& \geq\left(16 \sin ^{4} \frac{\pi}{2 T}+\varepsilon_{0}\right)|u|-a-b \varepsilon|u|, \quad \forall|u|>X_{1} .
\end{aligned}
$$

Now we choose $c_{1}=\left(16 \sin ^{4} \frac{\pi}{2 T}+\varepsilon_{0}-b \varepsilon\right) X_{1}+\max _{t \in \mathbb{T}_{2}^{T},|u| \leq X_{1}}|f(t, u)|$ and $k^{*}=\max _{|u| \leq X_{1}} k(u)$. Then we have

$$
\begin{aligned}
f(t, u) & \geq\left(16 \sin ^{4} \frac{\pi}{2 T}+\varepsilon_{0}-b \varepsilon\right)|u|-a-c_{1} \\
& =\left(16 \sin ^{4} \frac{\pi}{2 T}+\varepsilon_{0}-b \varepsilon\right)|u|-c_{2}, \quad \forall t \in \mathbb{T}_{2}^{T}, u \in \mathbb{R},
\end{aligned}
$$


where $c_{2}=c_{1}+a$. Note that $\varepsilon$ can be chosen arbitrarily small, and we let

$$
\begin{aligned}
R> & \max \left\{\frac{\left(c_{2}+b k^{*}\right)\left[\left(\varepsilon_{0}-b \varepsilon\right) \sum_{s=2}^{T} H(s, s) \sum_{j=2}^{T} H(s, j)+\left(16 \sin ^{4} \frac{\pi}{2 T}+\varepsilon_{0}-b \varepsilon\right) \sum_{s=2}^{T} \sum_{j=2}^{T} H(s, j)\right]}{\varepsilon_{0}-b \varepsilon-b \varepsilon\left[\left(\varepsilon_{0}-b \varepsilon\right) \sum_{s=2}^{T} H(s, s) \sum_{j=2}^{T} H(s, j)+\left(16 \sin ^{4} \frac{\pi}{2 T}+\varepsilon_{0}-b \varepsilon\right) \sum_{s=2}^{T} \sum_{j=2}^{T} H(s, j)\right]},\right. \\
& \left.\frac{\sum_{s=2}^{T} H(s, s) \sum_{j=2}^{T} H(s, j)\left(c_{2}+b k^{*}\right)}{1-b \varepsilon \sum_{s=2}^{T} H(s, s) \sum_{j=2}^{T} H(s, j)}, 0\right\} .
\end{aligned}
$$

Now we prove that

$$
u-A u \neq \mu \varphi_{0}, \quad \forall u \in \partial B_{R}, \mu \geq 0 .
$$

From (2.4) and Lemma 2.2, we have $\varphi_{0}=16 \sin ^{4} \frac{\pi}{2 T} L \varphi_{0} \in P_{0}$. Indeed, if (3.5) isn't true, then there exist $u_{0} \in \partial B_{R}$ and $\mu_{0}>0$ such that

$$
u_{0}-A u_{0}=\mu_{0} \varphi_{0}
$$

Let $\tilde{u}(t)=\sum_{s=2}^{T} H(t, s) \sum_{j=2}^{T} H(s, j)\left(a+b k\left(u_{0}\right)+c_{1}\right)$. Then

$$
\begin{aligned}
\tilde{u}(t) & \leq \sum_{s=2}^{T} H(t, s) \sum_{j=2}^{T} H(s, j)\left(c_{2}+b \varepsilon\left|u_{0}\right|+b k^{*}\right) \\
& \leq \sum_{s=2}^{T} H(s, s) \sum_{j=2}^{T} H(s, j)\left(c_{2}+b \varepsilon\left\|u_{0}\right\|+b k^{*}\right) .
\end{aligned}
$$

Therefore,

$$
\|\tilde{u}\| \leq \sum_{s=2}^{T} H(s, s) \sum_{j=2}^{T} H(s, j)\left(c_{2}+b \varepsilon R+b k^{*}\right) .
$$

Then from $L(P) \subset P_{0}, \varphi_{0} \in P_{0}$, and

$$
\begin{aligned}
u_{0}(t)+\tilde{u}(t) & =\tilde{u}(t)+\left(A u_{0}\right)(t)+\mu_{0} \varphi_{0}(t) \\
& =\sum_{s=2}^{T} H(t, s) \sum_{j=2}^{T} H(s, j)\left(f\left(j, u_{0}(j)\right)+b k\left(u_{0}(j)\right)+a+c_{1}\right)+\mu_{0} \varphi_{0}(t),
\end{aligned}
$$

we have

$$
u_{0}+\tilde{u} \in P_{0}
$$

As a result, we obtain

$$
\begin{aligned}
& \left(A u_{0}\right)(t)+\tilde{u}(t) \\
& \quad=\sum_{s=2}^{T} H(t, s) \sum_{j=2}^{T} H(s, j)\left(f\left(j, u_{0}(j)\right)+b k\left(u_{0}(j)\right)+c_{2}\right)
\end{aligned}
$$




$$
\begin{aligned}
& \geq \sum_{s=2}^{T} H(t, s) \sum_{j=2}^{T} H(s, j)\left(\left(16 \sin ^{4} \frac{\pi}{2 T}+\varepsilon_{0}-b \varepsilon\right)\left|u_{0}(j)\right|-c_{2}+b k\left(u_{0}(j)\right)+c_{2}\right) \\
& \geq\left(16 \sin ^{4} \frac{\pi}{2 T}+\varepsilon_{0}-b \varepsilon\right) \sum_{s=2}^{T} H(t, s) \sum_{j=2}^{T} H(s, j)\left|u_{0}(j)\right| \\
& \geq\left(16 \sin ^{4} \frac{\pi}{2 T}+\varepsilon_{0}-b \varepsilon\right) \sum_{s=2}^{T} H(t, s) \sum_{j=2}^{T} H(s, j) u_{0}(j) .
\end{aligned}
$$

On the other hand, from the definition of $L$, we get

$$
\begin{array}{r}
\left(16 \sin ^{4} \frac{\pi}{2 T}+\varepsilon_{0}-b \varepsilon\right) \sum_{s=2}^{T} H(t, s) \sum_{j=2}^{T} H(s, j) u_{0}(j) \\
=16 \sin ^{4} \frac{\pi}{2 T} \sum_{s=2}^{T} H(t, s) \sum_{j=2}^{T} H(s, j)\left(u_{0}(j)+\tilde{u}(j)\right) \\
-16 \sin ^{4} \frac{\pi}{2 T} \sum_{s=2}^{T} H(t, s) \sum_{j=2}^{T} H(s, j) \tilde{u}(j) \\
+\left(\varepsilon_{0}-b \varepsilon\right) \sum_{s=2}^{T} H(t, s) \sum_{j=2}^{T} H(s, j) u_{0}(j) \\
\geq 16 \sin ^{4} \frac{\pi}{2 T} \sum_{s=2}^{T} H(t, s) \sum_{j=2}^{T} H(s, j)\left(u_{0}(j)+\tilde{u}(j)\right) ;
\end{array}
$$

in order to obtain the above inequality, we prove that

$$
\begin{aligned}
& -16 \sin ^{4} \frac{\pi}{2 T} \sum_{s=2}^{T} H(t, s) \sum_{j=2}^{T} H(s, j) \tilde{u}(j) \\
& +\left(\varepsilon_{0}-b \varepsilon\right) \sum_{s=2}^{T} H(t, s) \sum_{j=2}^{T} H(s, j) u_{0}(j) \geq 0 .
\end{aligned}
$$

Indeed, since $u_{0}+\tilde{u} \in P_{0}$, we have $u_{0}(t)+\tilde{u}(t) \geq e(t)\left\|u_{0}+\tilde{u}\right\| \geq e(t)\left(\left\|u_{0}\right\|-\|\tilde{u}\|\right)$. Note that $H(t, s)$ vanishes at $t=1$ and $t=T+1, H(t, s)$ is symmetric on $\mathbb{T}_{2}^{T}$, i.e., $H(t, s)=H(s, t)$. Then

$$
\begin{aligned}
\left(\varepsilon_{0}-b \varepsilon\right) & \sum_{s=2}^{T} H(t, s) \sum_{j=2}^{T} H(s, j)\left(\tilde{u}(j)+u_{0}(j)\right) \\
& -\left(16 \sin ^{4} \frac{\pi}{2 T}+\varepsilon_{0}-b \varepsilon\right) \sum_{s=2}^{T} H(t, s) \sum_{j=2}^{T} H(s, j) \tilde{u}(j) \\
\geq & \left(\varepsilon_{0}-b \varepsilon\right)(R-\|\tilde{u}\|) \sum_{s=2}^{T} H(t, s) \sum_{j=2}^{T} H(s, j) e(j) \\
& -\left(16 \sin ^{4} \frac{\pi}{2 T}+\varepsilon_{0}-b \varepsilon\right) \sum_{s=2}^{T} H(t, s)
\end{aligned}
$$




$$
\begin{aligned}
& \quad \times \sum_{j=2}^{T} H(s, j) e(j)\left(\sum_{s=2}^{T} \sum_{j=2}^{T} H(s, j)\left(c_{2}+b \varepsilon R+b k^{*}\right)\right) \\
& \geq 0 .
\end{aligned}
$$

Combining (3.8), (3.9) and (3.10), we have

$$
\begin{aligned}
\left(A u_{0}\right)(t)+\tilde{u}(t) & \geq 16 \sin ^{4} \frac{\pi}{2 T} \sum_{s=2}^{T} H(t, s) \sum_{j=2}^{T} H(s, j)\left(u_{0}(j)+\tilde{u}(j)\right) \\
& =16 \sin ^{4} \frac{\pi}{2 T}\left(L\left(u_{0}+\tilde{u}\right)\right)(t) .
\end{aligned}
$$

Using (3.6) we obtain

$$
u_{0}+\tilde{u}=A u_{0}+\tilde{u}+\mu_{0} \varphi_{0} \geq 16 \sin ^{4} \frac{\pi}{2 T} L\left(u_{0}+\tilde{u}\right)+\mu_{0} \varphi_{0} \geq \mu_{0} \varphi_{0} .
$$

Define

$$
\mu^{*}=\sup \left\{\mu>0: u_{0}+\tilde{u} \geq \mu \varphi_{0}\right\}
$$

Note that $\mu_{0} \in\left\{\mu>0: u_{0}+\tilde{u} \geq \mu \varphi_{0}\right\}$, and then $\mu^{*} \geq \mu_{0}, u_{0}+\tilde{u} \geq \mu^{*} \varphi_{0}$. From (2.4) we have

$$
16 \sin ^{4} \frac{\pi}{2 T} L\left(u_{0}+\tilde{u}\right) \geq \mu^{*} 16 \sin ^{4} \frac{\pi}{2 T} L \varphi_{0}=\mu^{*} \varphi_{0},
$$

and hence

$$
u_{0}+\tilde{u} \geq 16 \sin ^{4} \frac{\pi}{2 T} L\left(u_{0}+\tilde{u}\right)+\mu_{0} \varphi_{0} \geq\left(\mu_{0}+\mu^{*}\right) \varphi_{0},
$$

which contradicts the definition of $\mu^{*}$. Therefore, (3.5) holds, and from Lemma 2.3 we obtain

$$
\operatorname{deg}\left(I-A, B_{R}, 0\right)=0
$$

On the other hand, from (H4), there exist $\varepsilon_{1} \in\left(0,16 \sin ^{4} \frac{\pi}{2 T}\right)$ and $r \in(0, R)$ such that

$$
|f(t, u)| \leq\left(16 \sin ^{4} \frac{\pi}{2 T}-\varepsilon_{1}\right)|u|, \quad \forall t \in \mathbb{T}_{2}^{T},|u|<r .
$$

Now for this $r$, we show that

$$
A u \neq \mu u, \quad u \in \partial B_{r}, \mu \geq 1 .
$$

Otherwise, there would exist $u_{1} \in \partial B_{r}, \mu_{1} \geq 1$ such that

$$
\begin{aligned}
\left|u_{1}(t)\right| & =\frac{1}{\mu_{1}}\left|\left(A u_{1}\right)(t)\right| \leq\left|\left(A u_{1}\right)(t)\right| \\
& =\left|\sum_{s=2}^{T} H(t, s) \sum_{j=2}^{T} H(s, j) f\left(j, u_{1}(j)\right)\right|
\end{aligned}
$$




$$
\begin{aligned}
& \leq \sum_{s=2}^{T} H(t, s) \sum_{j=2}^{T} H(s, j)\left|f\left(j, u_{1}(j)\right)\right| \\
& \leq\left(16 \sin ^{4} \frac{\pi}{2 T}-\varepsilon_{1}\right) \sum_{s=2}^{T} H(t, s) \sum_{j=2}^{T} H(s, j)\left|u_{1}(j)\right| .
\end{aligned}
$$

Multiplying both sides of the above inequality by $\sin \frac{\pi(t-1)}{T}$, then summing from 2 to $T$, and using (2.5), we obtain

$$
\begin{aligned}
& \sum_{t=2}^{T}\left|u_{1}(t)\right| \sin \frac{\pi(t-1)}{T} \\
& \quad \leq\left(16 \sin ^{4} \frac{\pi}{2 T}-\varepsilon_{1}\right) \sum_{t=2}^{T}\left[\sum_{s=2}^{T} H(t, s) \sum_{j=2}^{T} H(s, j)\left|u_{1}(j)\right|\right] \sin \frac{\pi(t-1)}{T} \\
& \quad=\frac{16 \sin ^{4} \frac{\pi}{2 T}-\varepsilon_{1}}{16 \sin ^{4} \frac{\pi}{2 T}} \sum_{t=2}^{T}\left|u_{1}(t)\right| \sin \frac{\pi(t-1)}{T} .
\end{aligned}
$$

This implies that $\sum_{t=2}^{T}\left|u_{1}(t)\right| \sin \frac{\pi(t-1)}{T}=0$, and whence $u_{1}(t) \equiv 0$, which contradicts $u_{1} \in$ $\partial B_{r}$. Hence, (3.15) holds, and from Lemma 2.4 we obtain

$$
\operatorname{deg}\left(I-A, B_{r}, 0\right)=1 .
$$

This, together with (3.13), implies that

$$
\operatorname{deg}\left(I-A, B_{R} \backslash \bar{B}_{r}, 0\right)=\operatorname{deg}\left(I-A, B_{R}, 0\right)-\operatorname{deg}\left(I-A, B_{r}, 0\right)=-1 .
$$

Therefore, the operator $A$ has at least one fixed point in $B_{R} \backslash \bar{B}_{r}$, and (1.1) has at least one nontrivial solution. This completes the proof.

Theorem 3.2 Suppose that (H5)-(H6) hold. Then (1.1) has at least one nontrivial solution.

Proof From (H5), there are $\varepsilon_{2} \in\left(0,16 \sin ^{4} \frac{\pi}{2 T}\right)$ and $r>0$ such that

$$
f(t, u) \geq\left(16 \sin ^{4} \frac{\pi}{2 T}+\varepsilon_{2}\right) u, \quad \forall u \in[0, r], t \in \mathbb{T}_{2}^{T},
$$

and

$$
f(t, u) \geq\left(16 \sin ^{4} \frac{\pi}{2 T}-\varepsilon_{2}\right) u, \quad \forall u \in[-r, 0], t \in \mathbb{T}_{2}^{T} .
$$

The above two inequalities enable us to obtain

$$
\begin{aligned}
& f(t, u) \geq\left(16 \sin ^{4} \frac{\pi}{2 T}+\varepsilon_{2}\right) u, \quad \forall u \in[-r, r], t \in \mathbb{T}_{2}^{T}, \\
& f(t, u) \geq\left(16 \sin ^{4} \frac{\pi}{2 T}-\varepsilon_{2}\right) u, \quad \forall u \in[-r, r], t \in \mathbb{T}_{2}^{T} .
\end{aligned}
$$


Define a cone $P_{1}$ as follows:

$$
P_{1}=\left\{u \in P: \sum_{t=2}^{T} u(t) \sin \frac{\pi(t-1)}{T} \geq \delta\|u\|\right\}
$$

where $\delta=\sum_{t=2}^{T} e(t) \sin \frac{\pi(t-1)}{T}$. Then we claim

$$
L(P) \subset P_{1}
$$

Indeed, for $u \in P$, from Lemma 2.1 we have

$$
\begin{aligned}
\sum_{t=2}^{T}(L u)(t) \sin \frac{\pi(t-1)}{T} & =\sum_{t=2}^{T} \sum_{s=2}^{T} H(t, s) \sum_{j=2}^{T} H(s, j) u(j) \sin \frac{\pi(t-1)}{T} \\
& \geq \sum_{t=2}^{T} \sum_{s=2}^{T} e(t) H(\tau, s) \sum_{j=2}^{T} H(s, j) u(j) \sin \frac{\pi(t-1)}{T} \\
& =\delta(L u)(\tau), \quad \forall \tau \in \mathbb{T}_{2}^{T},
\end{aligned}
$$

and thus

$$
\sum_{t=2}^{T}(L u)(t) \sin \frac{\pi(t-1)}{T} \geq \delta\|L u\|
$$

Moreover, $\varphi_{0} \in P_{1}$ since $\varphi_{0}=16 \sin ^{4} \frac{\pi}{2 T} L \varphi_{0} \in P_{1}$. Now we claim that

$$
u-A u \neq \mu \varphi_{0}, \quad \forall u \in \partial B_{r}, \mu \geq 0 .
$$

If the claim is false, then there exist $u_{2} \in \partial B_{r}$ and $\mu_{2} \geq 0$ such that

$$
u_{2}-A u_{2}=\mu_{2} \varphi_{0}
$$

From (3.17) we have $A u_{2} \geq\left(16 \sin ^{4} \frac{\pi}{2 T}+\varepsilon_{2}\right) L u_{2}$ and so $u_{2} \geq\left(16 \sin ^{4} \frac{\pi}{2 T}+\varepsilon_{2}\right) L u_{2}$, i.e.,

$$
u_{2}(t) \geq\left(16 \sin ^{4} \frac{\pi}{2 T}+\varepsilon_{2}\right) \sum_{s=2}^{T} H(t, s) \sum_{j=2}^{T} H(s, j) u_{2}(j)
$$

Multiplying both sides of the above inequality by $\sin \frac{\pi(t-1)}{T}$, then summing from 2 to $T$, and using (2.5), we obtain

$$
\begin{aligned}
& \sum_{t=2}^{T} u_{2}(t) \sin \frac{\pi(t-1)}{T} \\
& \quad \geq\left(16 \sin ^{4} \frac{\pi}{2 T}+\varepsilon_{2}\right) \sum_{t=2}^{T}\left[\sum_{s=2}^{T} H(t, s) \sum_{j=2}^{T} H(s, j) u_{2}(j)\right] \sin \frac{\pi(t-1)}{T} \\
& \quad=\frac{16 \sin ^{4} \frac{\pi}{2 T}+\varepsilon_{2}}{16 \sin ^{4} \frac{\pi}{2 T}} \sum_{t=2}^{T} u_{2}(t) \sin \frac{\pi(t-1)}{T}
\end{aligned}
$$


which implies that

$$
\sum_{t=2}^{T} u_{2}(t) \sin \frac{\pi(t-1)}{T} \leq 0
$$

On the other hand, from (3.21) we have

$$
\begin{aligned}
u_{2}(t) & -\left(16 \sin ^{4} \frac{\pi}{2 T}-\varepsilon_{2}\right)\left(L u_{2}\right)(t) \\
= & \left(A u_{2}\right)(t)-\left(16 \sin ^{4} \frac{\pi}{2 T}-\varepsilon_{2}\right)\left(L u_{2}\right)(t)+\mu_{2} \varphi_{0}(t) \\
& =\sum_{s=2}^{T} H(t, s) \sum_{j=2}^{T} H(s, j)\left[f\left(j, u_{2}(j)\right)-\left(16 \sin ^{4} \frac{\pi}{2 T}-\varepsilon_{2}\right) u_{2}(j)\right]+\mu_{2} \varphi_{0}(t) .
\end{aligned}
$$

Then (3.18), (3.19) and $\varphi_{0} \in P_{1}$ enable us to find $u_{2}-\left(16 \sin ^{4} \frac{\pi}{2 T}-\varepsilon_{2}\right) L u_{2} \in P_{1}$, and thus

$$
\begin{aligned}
\| u_{2} & -\left(16 \sin ^{4} \frac{\pi}{2 T}-\varepsilon_{2}\right) L u_{2} \| \\
\leq & \frac{1}{\delta} \sum_{t=2}^{T}\left[u_{2}(t)-\left(16 \sin ^{4} \frac{\pi}{2 T}-\varepsilon_{2}\right)\left(L u_{2}\right)(t)\right] \sin \frac{\pi(t-1)}{T} \\
= & \frac{\varepsilon_{2}}{\delta 16 \sin ^{4} \frac{\pi}{2 T}} \sum_{t=2}^{T} u_{2}(t) \sin \frac{\pi(t-1)}{T} \leq 0 .
\end{aligned}
$$

Note that $\left(16 \sin ^{4} \frac{\pi}{2 T}-\varepsilon_{2}\right) r(L)<1$, where $r(L)$ is the spectral radius of $L$. Hence, we have $u_{2}=0$, contradicting $u_{2} \in \partial B_{r}$. This implies that (3.20) holds, and from Lemma 2.3 we have

$$
\operatorname{deg}\left(I-A, B_{r}, 0\right)=0
$$

On the other hand, from (H6) there exist $\varepsilon_{3} \in\left(0,16 \sin ^{4} \frac{\pi}{2 T}\right)$ and $c_{3}>0$ such that

$$
|f(t, u)| \leq\left(16 \sin ^{4} \frac{\pi}{2 T}-\varepsilon_{3}\right)|u|+c_{3}, \quad \forall t \in \mathbb{T}_{2}^{T}, u \in \mathbb{R} .
$$

Let $\mathcal{M}=\{u \in E: u=\lambda A u, \lambda \in[0,1]\}$. Then we prove that $\mathcal{M}$ is bounded in $E$. If $u \in \mathcal{M}$, then from (3.24) we have

$$
\begin{aligned}
|u(t)| & =\lambda|(A u)(t)| \leq \sum_{s=2}^{T} H(t, s) \sum_{j=2}^{T} H(s, j)|f(j, u(j))| \\
& \leq \sum_{s=2}^{T} H(t, s) \sum_{j=2}^{T} H(s, j)\left[\left(16 \sin ^{4} \frac{\pi}{2 T}-\varepsilon_{3}\right)|u(j)|+c_{3}\right] .
\end{aligned}
$$

Multiplying both sides of the above inequality by $\sin \frac{\pi(t-1)}{T}$, then summing from 2 to $T$, and using (2.5), we obtain

$$
\sum_{t=2}^{T}|u(t)| \sin \frac{\pi(t-1)}{T} \leq \frac{1}{16 \sin ^{4} \frac{\pi}{2 T}} \sum_{t=2}^{T}\left[\left(16 \sin ^{4} \frac{\pi}{2 T}-\varepsilon_{3}\right)|u(t)|+c_{3}\right] \sin \frac{\pi(t-1)}{T},
$$


and then

$$
\sum_{t=2}^{T}|u(t)| \sin \frac{\pi(t-1)}{T} \leq c_{3} \varepsilon_{3}^{-1} \sum_{t=2}^{T} \sin \frac{\pi(t-1)}{T}
$$

We know that there is a $t_{0} \in \mathbb{T}_{2}^{T}$ such that $\|u\|=\left|u\left(t_{0}\right)\right|$, and thus

$$
\left|u\left(t_{0}\right)\right| \sin \frac{\pi\left(t_{0}-1\right)}{T} \leq \sum_{t=2}^{T}|u(t)| \sin \frac{\pi(t-1)}{T} .
$$

This implies that

$$
\|u\| \leq c_{3} \varepsilon_{3}^{-1} \sin ^{-1} \frac{\pi\left(t_{0}-1\right)}{T} \sum_{t=2}^{T} \sin \frac{\pi(t-1)}{T},
$$

proving the boundedness of $\mathcal{M}$. Choose $R>\max \left\{\sup _{u \in \mathcal{M}}\|u\|, r\right\}$ ( $r$ is defined by (3.17)), then

$$
\lambda A u \neq u, \quad u \in \partial B_{R}, \lambda \in[0,1] .
$$

Lemma 2.4 implies that

$$
\operatorname{deg}\left(I-A, B_{R}, 0\right)=1 .
$$

This, together with (3.23), implies that

$$
\operatorname{deg}\left(I-A, B_{R} \backslash \bar{B}_{r}, 0\right)=\operatorname{deg}\left(I-A, B_{R}, 0\right)-\operatorname{deg}\left(I-A, B_{r}, 0\right)=1 .
$$

Therefore, the operator $A$ has at least one fixed point in $B_{R} \backslash \bar{B}_{r}$, and (1.1) has at least one nontrivial solution. This completes the proof.

Example 3.3 Let $f(t, x)=a|x|-b k(x), k(x)=\ln (|x|+1), x \in \mathbb{R}$, where $a \in\left(16 \sin ^{4} \frac{\pi}{2 T},+\infty\right)$ and $b \in\left(0, a+16 \sin ^{4} \frac{\pi}{2 T}\right)$. Then $\lim _{|x| \rightarrow+\infty} \frac{k(x)}{|x|}=0$, and $\lim _{|x| \rightarrow+\infty} \frac{a|x|-b \ln (|x|+1)}{|x|}=a>$ $16 \sin ^{4} \frac{\pi}{2 T}, \lim _{|x| \rightarrow 0} \frac{|a| x|-b \ln (|x|+1)|}{|x|}=|a-b|<16 \sin ^{4} \frac{\pi}{2 T}$. Therefore, (H1)-(H4) hold.

Example 3.4 Let $f(t, x)=\left\{\begin{array}{ll}a x+b \sin x, & x \geq 0, \\ a x-b e^{x}+b, & x \leq 0,\end{array}\right.$ where $a, b>0$ with $a<16 \sin ^{4} \frac{\pi}{2 T}, a+b>$ $16 \sin ^{4} \frac{\pi}{2 T}$ and $a-b<16 \sin ^{4} \frac{\pi}{2 T}$. Then $\lim _{x \rightarrow 0^{+}} \frac{a x+b \sin x}{x}=a+b, \lim _{x \rightarrow 0^{-}} \frac{a x-b e^{x}+b}{x}=a-b$, $\lim _{x \rightarrow+\infty}\left|\frac{a x+b \sin x}{x}\right|=a$, and $\lim _{x \rightarrow-\infty}\left|\frac{a x-b e^{x}+b}{x}\right|=a$. Therefore, (H5)-(H6) hold.

\section{Conclusions}

In this paper, we established the existence of nontrivial solutions for the boundary value problems of the fourth order difference equation (1.1) with sign-changing nonlinearity using the topological degree theory. Under some conditions concerning the first eigenvalue corresponding to the relevant linear problem, the results here improve and generalize those obtained in [1-11]. 


\section{Acknowledgements}

The authors are grateful to the referees for their valuable suggestions and comments.

\section{Funding}

This work is supported by Natural Science Foundation of Shandong Province (ZR2018MA009, ZR2015AM014).

Availability of data and materials

Not applicable.

\section{Competing interests}

The authors declare that they have no competing interests.

\section{Authors' contributions}

This paper is the result of joint work of all authors who contributed equally to the final version of this paper. All authors read and approved the final manuscript.

\section{Author details}

'School of Mathematics, Qilu Normal University, Jinan, China. ${ }^{2}$ School of Mathematics, Statistics and Applied Mathematics, National University of Ireland, Galway, Ireland. ${ }^{3}$ College of Mathematics and System Sciences, Shandong University of Science and Technology, Qingdao, China.

\section{Publisher's Note}

Springer Nature remains neutral with regard to jurisdictional claims in published maps and institutional affiliations.

Received: 17 August 2018 Accepted: 7 October 2018 Published online: 11 October 2018

\section{References}

1. Goodrich, C.S.: On discrete sequential fractional boundary value problems. J. Math. Anal. Appl. 385, 111-124 (2012)

2. LV, Z., Gong, Y., Chen, Y.: Multiplicity and uniqueness for a class of discrete fractional boundary value problems. Appl. Math. 59(6), 673-695 (2014)

3. Ma, R., Xu, Y.: Existence of positive solution for nonlinear fourth-order difference equations. Comput. Math. Appl. 59, 3770-3777 (2010)

4. Xu, J.: Positive solutions for a fourth order discrete $p$-Laplacian boundary value problem. Math. Methods Appl. Sci. 36, 2467-2475 (2013)

5. Rehman, M., Iqbal, F., Seemab, A.: On existence of positive solutions for a class of discrete fractional boundary value problems. Positivity 21, 1173-1187 (2017)

6. Bai, D., Henderson, J., Zeng, Y.: Positive solutions of discrete Neumann boundary value problems with sign-changing nonlinearities. Bound. Value Probl. 2015, Article ID 231 (2015)

7. Goodrich, C.S.: On a first-order semipositone discrete fractional boundary value problem. Arch. Math. 99, 509-518 (2012)

8. Goodrich, C.S.: On semipositone discrete fractional boundary value problems with non-local boundary conditions. J. Differ. Equ. Appl. 19(11), 1758-1780 (2013)

9. Dahal, R., Duncan, D., Goodrich, C.S.: Systems of semipositone discrete fractional boundary value problems. J. Differ. Equ. Appl. 20(3), 473-491 (2014)

10. Xu, J., O'Regan, D.: Existence and uniqueness of solutions for a first-order discrete fractional boundary value problem. Rev. R. Acad. Cienc. Exactas Fís. Nat., Ser. A Mat. (in press)

11. Xu, J., Goodrich, C.S., Cui, Y.: Positive solutions for a system of first-order discrete fractional boundary value problems with semipositone nonlinearities. Rev. R. Acad. Cienc. Exactas Fís. Nat., Ser. A Mat. 112(4), 1005-1016 (2018)

12. Wang, Y., Liu, L.: Positive solutions for a class of fractional infinite-point boundary value problems. Bound. Value Probl. 2018, Article ID 118 (2018)

13. Wang, Y., Liu, L., Wu, Y.: Positive solutions for a class of fractional boundary value problem with changing sign nonlinearity. Nonlinear Anal., Theory Methods Appl. 74(17), 6434-6441 (2011)

14. Wang, Y., Liu, L., Zhang, X., Wu, Y.: Positive solutions of an abstract fractional semipositone differential system model for bioprocesses of HIV infection. Appl. Math. Comput. 258, 312-324 (2015)

15. Zhang, X., Liu, L., Zou, Y.: Fixed-point theorems for systems of operator equations and their applications to the fractional differential equations. J. Funct. Spaces 2018, Article ID 7469868 (2018)

16. Hao, X., Wang, H., Liu, L., Cui, Y.: Positive solutions for a system of nonlinear fractional nonlocal boundary value problems with parameters and p-Laplacian operator. Bound. Value Probl. 2017, Article ID 182 (2017)

17. Zhao, Z: Positive solutions of semi-positone Hammerstein integral equations and applications. Appl. Math. Comput. 219(5), 2789-2797 (2012)

18. Liu, J., Zhao, Z:: Multiple positive solutions for second-order three-point boundary-value problems with sign changing nonlinearities. Electron. J. Differ. Equ. 2012, 152 (2012)

19. Zhao, Z: Existence of positive solutions for 2 thth-order singular semipositone differential equations with Sturm-Liouville boundary conditions. Nonlinear Anal., Theory Methods Appl. 72(3-4), 1348-1357 (2010)

20. Lin, X., Zhao, Z: Sign-changing solution for a third-order boundary-value problem in ordered Banach space with lattice structure. Bound. Value Probl. 2014, Article ID 132 (2014)

21. Wu, Y., Zhao, Z.: Positive solutions for third-order boundary value problems with change of signs. Appl. Math. Comput. 218(6), 2744-2749 (2011)

22. Zhang, K:: On a sign-changing solution for some fractional differential equations. Bound. Value Probl. 2017, Article ID 59 (2017) 
23. Zhang, K:: Nontrivial solutions of fourth-order singular boundary value problems with sign-changing nonlinear terms. Topol. Methods Nonlinear Anal. 40(1), 53-70 (2012)

24. Fan, W., Hao, X., Liu, L., Wu, Y.: Nontrivial solutions of singular fourth-order Sturm-Liouville boundary value problems with a sign-changing nonlinear term. Appl. Math. Comput. 217(15), 6700-6708 (2011)

25. Hao, X., Zuo, M., Liu, L.: Multiple positive solutions for a system of impulsive integral boundary value problems with sign-changing nonlinearities. Appl. Math. Lett. 82, 24-31 (2018)

26. Liu, L., Liu, B., Wu, Y.: Nontrivial solutions for higher-order $m$-point boundary value problem with a sign-changing nonlinear term. Appl. Math. Comput. 217(8), 3792-3800 (2010)

27. Guo, Y.: Nontrivial solutions for boundary-value problems of nonlinear fractional differential equations. Bull. Korean Math. Soc. 47(1), 81-87 (2010)

28. Guo, Y: Nontrivial periodic solutions of nonlinear functional differential systems with feedback control. Turk. J. Math. 34(1), 35-44 (2010)

29. Guo, Y.: Positive solutions of second-order semipositone singular three-point boundary value problems. Electron. J. Qual. Theory Differ. Equ. 2009, 5 (2009)

30. Qiu, X., Xu, J., O'Regan, D., Cui, Y.: Positive solutions for a system of nonlinear semipositone boundary value problems with Riemann-Liouville fractional derivatives. J. Funct. Spaces 2018, Article ID 7351653 (2018)

31. Pu, R., Zhang, X., Cui, Y., Li, P., Wang, W.: Positive solutions for singular semipositone fractional differential equation subject to multipoint boundary conditions. J. Funct. Spaces 2017, Article ID 5892616 (2017)

32. Chen, C., Xu, J., O'Regan, D., Fu, Z.: Positive solutions for a system of semipositone fractional difference boundary value problems. J. Funct. Spaces 2018, Article ID 6835028 (2018)

33. Cheng, W., Xu, J., Cui, Y.: Positive solutions for a system of nonlinear semipositone fractional $q$-difference equations with q-integral boundary conditions. J. Nonlinear Sci. Appl. 10(8), 4430-4440 (2017)

34. Li, H., Sun, J.: Positive solutions of sublinear Sturm-Liouville problems with changing sign nonlinearity. Comput. Math. Appl. 58(9), 1808-1815 (2009)

35. Li, H., Sun, J.: Positive solutions of superlinear semipositone nonlinear boundary value problems. Comput. Math. Appl. 61(9), 2806-2815 (2011)

36. Wu, J., Zhang, X., Liu, L., Wu, Y., Cui, Y.: The convergence analysis and error estimation for unique solution of a p-Laplacian fractional differential equation with singular decreasing nonlinearity. Bound. Value Probl. 2018, Article ID $82(2018)$

37. Zuo, M., Hao, X., Liu, L., Cui, Y.: Existence results for impulsive fractional integro-differential equation of mixed type with constant coefficient and antiperiodic boundary conditions. Bound. Value Probl. 2017, Article ID 161 (2017)

38. Cui, Y.: Computation of topological degree in ordered Banach spaces with lattice structure and applications. Appl. Math. 58(6), 689-702 (2013)

39. Cui, Y., Ma, W., Sun, Q., Su, X.: New uniqueness results for boundary value problem of fractional differential equation. Nonlinear Anal., Model. Control 23(1), 31-39 (2018)

40. Cui, Y.: Uniqueness of solution for boundary value problems for fractional differential equations. Appl. Math. Lett. 51 , 48-54 (2016)

41. Zou, Y., He, G.: On the uniqueness of solutions for a class of fractional differential equations. Appl. Math. Lett. 74, 68-73 (2017)

42. Zhang, X., Liu, L., Wu, Y., Zou, Y.: Existence and uniqueness of solutions for systems of fractional differential equations with Riemann-Stieltjes integral boundary condition. Adv. Differ. Equ. 2018, Article ID 204 (2018)

43. Bai, Z., Dong, X., Yin, C.: Existence results for impulsive nonlinear fractional differential equation with mixed boundary conditions. Bound. Value Probl. 2016, Article ID 63 (2016)

44. Bai, Z., Zhang, Y.: Solvability of fractional three-point boundary value problems with nonlinear growth. Appl. Math. Comput. 218(5), 1719-1725 (2011)

45. Zhang, Y., Bai, Z., Feng, T.: Existence results for a coupled system of nonlinear fractional three-point boundary value problems at resonance. Comput. Math. Appl. 61(4), 1032-1047 (2011)

46. Guo, D., Lakshmikantham, V.: Nonlinear Problems in Abstract Cones. Academic Press, Orlando (1988)

\section{Submit your manuscript to a SpringerOpen ${ }^{\circ}$ journal and benefit from:}

- Convenient online submission

- Rigorous peer review

- Open access: articles freely available online

- High visibility within the field

- Retaining the copyright to your article

Submit your next manuscript at $\gg$ springeropen.com 